\title{
L'action collective et la durabilité des filières agricoles au Costa Rica
}

Collective action and sustainability of agricultural subsectors in Costa Rica

Élodie Maître d'Hôtel, Pierre-Marie Bosc et Johny Egg

\section{(2) OpenEdition}

Journals

Édition électronique

URL : http://journals.openedition.org/economierurale/631

DOI : $10.4000 /$ economierurale.631

ISSN : 2105-2581

Éditeur

Société Française d'Économie Rurale (SFER)

Édition imprimée

Date de publication : 1 juin 2008

Pagination : 123-135

ISSN : 0013-0559

Référence électronique

Élodie Maître d'Hôtel, Pierre-Marie Bosc et Johny Egg, "L'action collective et la durabilité des filières agricoles au Costa Rica », Économie rurale [En ligne], 303-304-305 | Janvier-juin 2008, mis en ligne le 01 juillet 2010, consulté le 01 mai 2019. URL : http://journals.openedition.org/economierurale/631 DOI : 10.4000/economierurale.631 


\title{
L'action collective et la durabilité des filières agricoles au Costa Rica
}

\author{
Élodie MAîTRE D'HÔTEL • SUPAGRO, CIRAD, UMR Marchés, Organisations, Institutions et \\ Stratégies d'Acteurs (MOÏSA)
}

Pierre-Marie BOSC • CIRAD, UMR (MOÏSA)

Johny EGG • INRA, UMR (MOÏSA)

\section{Introduction}

D ans les pays du Sud, les politiques économiques sont marquées de façon croissante par la libéralisation qui se caractérise à la fois par le retrait de l'État de l'appui à certains secteurs et par l'ouverture des marchés à la concurrence internationale. Le secteur agricole est particulièrement concerné : le démantèlement progressif des politiques agricoles et la globalisation des échanges commerciaux se traduisent par de profondes restructurations des territoires et de l'organisation économique des filières, dans lesquelles les acteurs privés sont amenés à occuper de nouvelles fonctions. De façon générale, l'adhésion de nombreux pays du Sud à l'Organisation mondiale du commerce (OMC) correspond à un approfondissement de la libéralisation de leurs économies. Une observation plus attentive révèle toutefois que la libéralisation recouvre différentes formes selon les contextes institutionnels dans lesquels elle s'opère.

Ainsi au Costa Rica, certaines filières agricoles ont été libéralisées brutalement quand d'autres filières bénéficiaient de mesures publiques d'appui pouvant aller jusqu'à freiner la mise en œuvre de la libéralisation. Dans cet article, nous nous appuyons sur les exemples contrastés des filières lait et haricot au Costa Rica. La filière lait se développe à l'abri d'une protection tarifaire élevée alors que la filière haricot est menacée dans son maintien par une baisse drastique de sa protec- tion tarifaire. Ces exemples vont nous permettre d'illustrer la façon dont l'appui public différencié se traduit au final par des conditions de durabilité spécifiques à chaque filière, du moins si l'on considère les composantes économiques, sociales et politiques de la durabilité. Nous montrons (i) que les différences d'appuis publics entre les filières peuvent s'expliquer par les spécificités de l'action collective sur le processus d'élaboration des politiques ; et (ii) que les conditions de réussite de l'action collective s'inscrivent dans des logiques de dépendance de sentier où les processus d'apprentissage jouent pleinement. Pour ce faire, nous analysons la façon dont les organisations professionnelles des filières lait et haricot interviennent dans le processus de négociation des droits de douane. Nous mettons en évidence des capacités différentes d'influence sur le processus politique : négociées en 1995, les protections tarifaires du lait (haute) et du haricot (basse) divergent fortement ; par ailleurs, ces capacités $d$ 'action et d'influence des organisations ne sont pas fixées une fois pour toutes; elles évoluent comme le montre le relèvement de la protection tarifaire du haricot entre 1995 et 2005.

La première partie de l'article propose un cadrage théorique à partir des théories du changement institutionnel et de l'action collective. La deuxième partie mobilise ce cadre pour présenter à travers les deux études de cas (secteurs lait et haricot) les réactions des acteurs de ces filières face à un même 
macro changement institutionnel, l'adhésion du Costa Rica à l'OMC. Les différences de structuration organisationnelle des deux secteurs se traduisent par des comportements stratégiques différents de la part des organisations représentant leurs intérêts au sein de chaque secteur respectivement. Au final, ce sont des environnements économiques différents qui sont observés, plus ou moins favorables à la durabilité de chacune des filières. La troisième partie propose des éléments de réflexion quant aux conditions de réussite des processus d'action collective précédemment analysés.

\section{Le changement institutionnel comme cadre d'analyse}

L'étude porte sur la participation des organisations professionnelles agricoles au processus d'élaboration des politiques ${ }^{1}$. La littérature en économie néo-institutionnelle fournit des clés de lecture de l'action des organisations dans le champ politique. Nous choisissons de mobiliser plus particulièrement les travaux de Douglass North portant sur le changement institutionnel pour deux raisons principales : d'abord parce qu'ils sont notamment fondés sur un schéma d'interactions permanentes entre organisations et institutions ; ensuite parce qu'ils interrogent le passé de ces interactions. De façon très schématique, les institutions sont définies comme les « règles du jeu d'une société », et les organisations comme «les joueurs » (North, 1990). Ainsi, les actions mises en place par les organisations sont « une réponse à la structure institutionnelle des sociétés »; mais en retour, les organisations sont également «la cause principale de la transformation de la structure insti-

1. En économie, les théories de la capture de la réglementation (Stigler, 1971) et du public choice (Buchanan et Tullock, 1962) pourraient apporter des éclairages intéressants à notre question, mais elles n'accordent pas une place prépondérante à la définition des institutions et à leur mobilisation par les acteurs. tutionnelle » (North, 1993). Il n'y a donc pas de déterminisme structurel ${ }^{2}$ : dans un univers de règles du jeu donné, les organisations disposent d'une marge de liberté d'action qui explique leur capacité à modifier ces règles.

\section{Peu d'études empiriques sur les mécanismes du changement institutionnel}

En économie néo-institutionnelle, le rapport entre institutions et organisations est souvent analysé de façon statique : on étudie l'action des organisations dans un univers de règles du jeu donné (Cook et Chaddad, 2004 ; Ménard, 2000) ; de ce fait, les études portant sur le changement institutionnel visent de manière presque exclusive à en évaluer les effets (Reardon et Barret, 2000). Peu de travaux analysent le rapport institutions/organisations dans une perspective dynamique (Chabaud et $a l, 2005)$, ce qui fait que les mécanismes à l'œuvre dans le changement institutionnel sont encore aujourd'hui mal appréciés (Alston, 1996). Les pistes de recherche avancées par North sur le rôle des organisations dans la mise en place d'institutions nouvelles restent encore à explorer de manière empirique (Alston, op. cit. ; North, 1997 ; Shirley, 2004). Sur ces questions, quelques travaux ont été menés dans le champ des industries (Chabaud et al, op. cit. ; Glachant et Finon, 1998 ; Levy et Spiller, 1994), alors que très peu ont été réalisés dans le champ de l'agriculture. Une étude empirique est ici proposée pour comprendre les mécanismes à l'œuvre dans le changement institutionnel, en analysant la participation des organisations professionnelles agricoles à la définition de politiques économiques sectorielles.

2. Crozier et Friedberg critiquent vivement la théorie de la contingence structurelle et insistent sur le fait qu'aucun modèle d'adaptation des organisations à leur environnement n'est capable de rendre compte de la complexité des phénomènes qui guident la réaction des organisations en relation avec leur environnement (Crozier et Friedberg, 1977; Friedberg, 1993). 


\section{Mobilisation des approches liées à l'action collective}

La littérature sur le changement institutionnel ne fournit pas d'outils analytiques suffisamment précis pour étudier le comportement des organisations. Différents référentiels liés à l'action collective relient le comportement des organisations aux caractéristiques de ces organisations. En proposant une théorie générale de l'action collective, Olson (1978) insiste sur les critères de taille de l'organisation et d'homogénéité des membres comme déterminants de l'efficacité des organisations. Pour l'analyse spécifique des groupes de pression, il propose également deux sous-théories liées : - celle du sous-produit qui explique qu'un grand groupe puisse être actif s'il propose à ses membres des biens privés, en plus de la fonction de négociation de politique qui a une nature de bien collectif ;

- celle des intérêts spéciaux qui montre que les intérêts particuliers d'un groupe restreint tendent à triompher de ceux de l'ensemble des membres, souvent inactifs et peu organisés.

Dans le champ du management, et également dans celui de la sociologie, certains auteurs travaillent sur les ressources que les organisations mobilisent pour leur action (McCarthy et Zald, 1977), et sur la façon dont les différences de ressources entre organisations induisent des différences de résultats (Penrose, 1959; Wernerfelt, 1984).

\section{Méthodologie : choix du modèle, collecte et traitement des données}

Le cadre d'analyse présenté permet d'étudier la façon dont les organisations professionnelles agricoles participent au processus d'élaboration des politiques économiques, en reliant le comportement des organisations à certaines de leurs caractéristiques. Dans notre modèle d'analyse, nous appréhendons les caractéristiques des organisations par la notion de ressources, déclinées comme suit. - Ressources financières : l'importance, la stabilité, la spécificité et l'origine des finan- cements dont disposent les organisations professionnelles agricoles pour leurs actions en matière de négociation de politique sont évaluées.

- Ressources humaines : nous décrivons l'insertion des organisations dans les réseaux politiques (espaces de dialogue entre acteurs et de négociation avec le gouvernement), les relations interpersonnelles existant entre représentants des organisations et du gouvernement, mais également le soutien des organisations par leurs bases (taille des organisations, homogénéité des membres, mécanismes de concertation avec les membres).

- Ressources techniques : sont analysées à la fois les connaissances techniques dont disposent les organisations et leur capacité à élaborer des propositions politiques.

Sont également étudiées les trajectoires des organisations dans la mesure où elles traduisent des processus d'apprentissages cumulatifs à travers les expériences individuelles et collectives de leurs responsables et de leur personnel technique. Cet ensemble de compétences créées par l'expérience et la mobilisation de ressources diverses constitue en soi un capital que les organisations peuvent mobiliser en fonction des défis auxquels elles se trouvent confrontées, notamment dans le cadre de négociation de politiques publiques.

Cette étude empirique se fonde sur un travail d'enquêtes approfondi au Costa Rica. Entre mai 2003 et mai 2005, une centaine d'entretiens a été menée auprès de représentants d'organisations professionnelles (organisations dont les activités s'orientent principalement vers la défense politique des intérêts de certains groupes d'acteurs), d'organisations de producteurs agricoles (organisations dont les activités s'orientent principalement vers la transformation et la mise en marché de produits agricoles) mais aussi d'agents de l'administration publique, du gouvernement, de firmes privées, et de producteurs agricoles. Les entretiens, réalisés de façon ouverte ou 
semi-guidée, visaient la compréhension des trajectoires, des actions stratégiques et des caractéristiques de ces acteurs. Les données collectées ont été ponctuellement complétées par la revue de documents (archives des organisations, études universitaires, documents officiels, périodiques...). Le traitement des données s'est fait par des descriptions analytiques.

\section{Les filières lait et haricot au Costa Rica : des réponses contrastées à la libéralisation}

Au Costa Rica, il semble difficile de parler de libéralisation dans le cas de la filière lait malgré le cadre global de libéralisation de l'économie agricole adopté dès le milieu des années 1980. Cette filière, contrairement à d'autres, bénéficie encore actuellement d'une protection tarifaire importante. Quant à la filière haricot, produit de base de l'alimentation quotidienne, la libéralisation l'a profondément affectée notamment depuis 1995 à travers une baisse significative de la protection tarifaire dont elle pouvait se prévaloir avant l'adhésion du Costa Rica à l'OMC. Nous cherchons ici à expliquer le fait que le tournant de la libéralisation ait été négocié différemment pour chacune des filières et nous mettons l'accent sur la manière dont certaines organisations ont pu ou non investir les espaces politiques d'interactions avec le gouvernement. Les différences de situations économiques (Tableau 1) traduisent des différences notables en termes de capacités d'action de la part des organisations dans la négociation de nouvelles règles du jeu économique. Ces différences ont un impact assez direct sur les situations des agriculteurs, en conditionnant leurs possibilités à se maintenir ou non sur certains marchés mais aussi la durabilité de certains secteurs. Nous nous positionnons par rapport à une définition de l'agriculture durable, qui doit réunir des conditions à la fois économiques, sociales et environnementales (Landais, 1998) et donc s'évaluer par rapport à des échelles économiques, socio-territoriales, et agro-écologiques (Briquel et al, 2001). La composante environnementale de l'analyse est exclue afin de se concentrer sur les composantes sociales et économiques et une composante politique est ajoutée à l'appréciation de la durabilité des filières agricoles.

Entre 1995 et 2005, le secteur du lait est marqué par un mouvement de spécialisation et de concentration de la production au sein d'un nombre plus réduit d'unités de production, la production augmente et les exportations se développent. En revanche, à la fois la production et le nombre de producteurs de haricot s'effondrent dans la même période.

Le lait et le haricot sont deux productions destinées au marché intérieur. Nous focaliserons notre attention sur la négociation des droits de douane à l'importation. Le Costa Rica, en adhérant à l'OMC en 1994, s'engage à supprimer les barrières non tarifaires à l'importation : l'établissement de droits de douane devient alors le seul mécanisme autorisé de protection visà-vis des importations, du fait de l'interdiction des systèmes de normes de qualité, de quotas et de permis dont bénéficiaient jusque-là les produits agricoles. Face à cette situation, en 1995 le droit de douane

Tableau 1. Évolution des filières lait et haricot au Costa Rica (quelques indicateurs)

\begin{tabular}{l|r|r|r|r}
\hline & \multicolumn{2}{|c|}{ Lait } & \multicolumn{2}{c}{ Haricot } \\
\cline { 2 - 5 } & 1995 & 2005 & 1995 & 2005 \\
\hline Nombre de producteurs & 9047 & 5200 & 21500 & 6000 \\
\hline Volume de production (tonnes) & 590000 & 610000 & 25707 & 10503 \\
\hline Volume d'importation (tonnes) & - & - & - & 23154 \\
\hline Volume d'exportation (tonnes) & - & 57000 & - & - \\
\hline
\end{tabular}


Adhésion du Costa Rica à l'OMC (1994) : changement institutionnel macro-économique

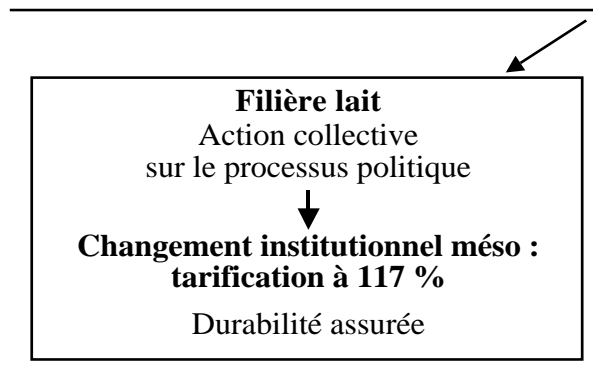

est fixé pour le lait à $117 \%$ (Arias, 2004), et pour le haricot à $1 \%$ (Salazar, 2000). Ni le haricot, ni le lait produits au Costa Rica ne sont compétitifs par rapport aux marchés internationaux, ils ne présentent donc pas d'avantages comparatifs pour l'économie nationale. A priori si l'on se fonde sur une analyse strictement économique selon la théorie des avantages compétitifs, le gouvernement n'a pas de raison au plan strictement économique de soutenir l'un plutôt que l'autre de ces produits.

Le changement institutionnel se produit à deux niveaux : d'abord au niveau macroéconomique (Figure 1), où l'adhésion du Costa Rica à l'OMC correspond à un choc économique que nous prenons comme une donnée ; ensuite, par répercussion, au niveau méso-économique (filières), du fait de la mobilisation des organisations professionnelles agricoles sur le processus de définition d'une politique de tarification.

\section{Le lait : une action concertée pour un niveau élevé de protection tarifaire}

Le lait est un produit historiquement destiné à la consommation nationale, mais qui, du fait d'un processus continu de développement, se positionne maintenant et depuis peu sur les marchés d'exportation en Amérique Centrale. La production de lait est le fait d'une base productive large de petits et moyens producteurs (SEPSA,

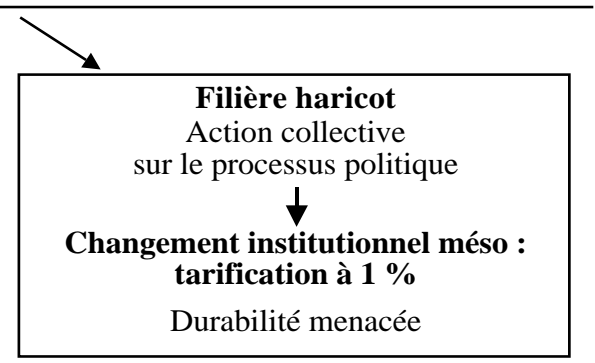

Source : conception des auteurs

2002) $)^{3}$. La majorité de la production est industrialisée $(70 \%)$, et les opérations de transformation et de commercialisation sont le fait principalement de structures coopératives qui détiennent $95 \%$ de parts de marché. La coopérative DosPinos, qui a intégré verticalement la filière, détient le quasi-monopole de la distribution du lait dans le pays (85\% de parts de marché). L'existence de structures de gouvernance quasi monopolistiques est à rapprocher de considérations techniques : le lait est un produit périssable qui nécessite des investissements particuliers pour sa production, sa transformation et son stockage (forte spécificité des actifs), et également une collecte fréquente (transactions fréquentes entre producteurs et coopératives). Les initiatives de DosPinos en terme de recherche et de vulgarisation ont fortement contribué au développement de la filière lait depuis les années 1950 (Melendez et Gonzalez, 1998) : la production et la productivité sont en augmentation croissante. Ces initiatives privées ont été en partie relayées par des programmes du ministère de l'Agriculture. Le fonctionnement de la filière laitière a traditionnellement été fortement régulé par l'État, par des systèmes de fixation des prix jusqu'en 1999 et de limitation des importations qui persistent encore.

3. Secrétariat exécutif de la planification sectorielle et agricole. 


\section{Défense des intérêts de la filière lait :} les acteurs

Les intérêts des producteurs et des industriels du lait sont représentés et défendus depuis 1965 par les activités de la Chambre nationale des producteurs et industriels du lait (CNPIL), qui constitue le principal espace de concertation entre les acteurs de la filière lait. L'activité économique étant le fait majoritairement de producteurs organisés en coopératives laitières, les intérêts économiques des producteurs et ceux des industriels sont en général convergents : en ce sens, les positions politiques adoptées par la CNPIL reflètent l'existence d'une vision de secteur. La coopérative DosPinos est partie intégrante de la CNPIL et suit de très près les activités de négociation politique. Le gouvernement, lorsqu'il souhaite discuter de la mise en place de certaines mesures spécifiques à la filière lait, convoque systématiquement des représentants de la CNPIL et la coopérative DosPinos. Ces mécanismes formels d'interactions se doublent de mécanismes informels de mobilisation du politique, du fait notamment d'une proximité sociale entre les acteurs. Certains dirigeants de la coopérative DosPinos ont des parcours politiques dans des postes de responsabilité élevée aux ministères des Finances ou de l'Économie. La filière lait se caractérise par une longue histoire d'interactions entre la CNPIL, avec notamment la coopérative DosPinos comme acteur dominant, et le gouvernement, qui a abouti à une réelle consolidation des mécanismes politiques. Leur mise en œuvre est facilitée par la visibilité des organisations actives politiquement, elle-même liée à une représentativité importante et à un poids économique important (le lait industrialisé correspondait à $12 \%$ de la valeur ajoutée agricole en 1995). La CNPIL est en 1995 la seule organisation de la filière lait à compter avec des experts en commerce international, donc à même de fournir une information technique de qualité (ou reconnue comme telle). Elle ne peut toutefois pas bénéficier de l'appui des producteurs de lait, qui ne saisissent alors pas l'enjeu des négociations politiques en cours. Globalement, en 1995, on peut considérer que la dotation en ressources de la CNPIL est satisfaisante, et se caractérise par :

- un niveau de technicité très élevé ;

- des capacités importantes de financement du fait notamment de la position hégémonique de DosPinos ;

- une insertion dans les réseaux politiques très poussée ;

- une expérience longue de relations avec le gouvernement.

L'appui des producteurs est le seul type de ressource qui fait défaut : la CNPIL cherche depuis à résoudre ces problèmes de faible mobilisation des producteurs en renforçant son offre de services aux producteurs (assistance technique, attribution de crédits), mais cette faible mobilisation s'explique aussi par la position dominante de l'agro-industrie au sein de la filière via la coopérative DosPinos.

\section{Défense des intérêts de la filière lait : \\ le processus}

Le processus de négociation politique qui a conduit à la fixation d'un droit de douane pour le lait par le ministère du Commerce extérieur comporte trois phases principales. Initialement, dans une première phase, les dirigeants de la CNPIL et de la coopérative DosPinos mènent conjointement un travail d'expertise, et évaluent à $117 \%$ le montant du droit de douane à l'importation pour compenser les distorsions internationales. Au cours d'une deuxième phase, ce modèle technique est discuté : à la fois en interne avec les producteurs des coopératives; et en externe avec des représentants du ministère de l'Agriculture et de la présidence de la République. Dans une troisième phase enfin, la CNPIL transmet la proposition discutée au ministère du Commerce extérieur et participe directement à la négociation, qui aboutit à l'établissement du droit de douane à $117 \%$ : le choix de 
politique publique correspond à l'évaluation technique du CNPIL. Actuellement, la participation des organisations agricoles de la filière lait au processus d'élaboration des politiques respecte les mêmes phases (élaboration, discussion et négociation). Le droit de douane a été ré-évalué une seule fois depuis, et reste à un niveau élevé qui protège la filière (Montero, 2004).

\section{Le haricot : des actions dispersées pour un niveau faible de protection}

Le haricot est un produit destiné à la consommation nationale. Sa production est le fait de nombreux petits producteurs qui produisent aussi d'autres denrées (Ruiz, 2002). En hausse continue jusqu'en 1994, la production connaît depuis une période de crise, marquée par un effondrement de la production nationale et une diminution importante du nombre de producteurs (Salazar, 2002). Jusqu'en 1994, l'État, en plus de politiques de subvention des prix, assurait intégralement les opérations de transformation (séchage) et de distribution de la production. Depuis le retrait de l'État, ce sont les entreprises privées (industriels) qui ont investi ces fonctions, en choisissant de s'approvisionner préférentiellement sur le marché international où les prix sont plus bas (les industriels importent environ $75 \%$ du haricot qu'ils distribuent). Les producteurs, fortement menacés par les importations, ont localement mis en place des organisations et cherché à négocier des contrats de vente avec les industriels : l'importance économique de ces organisations est toutefois réduite à $10 \%$ des parts de marché. D'un point de vue technique, le haricot est un produit qui se conserve facilement et n'exige pas d'investissements particuliers (ni pour sa production, ni pour son séchage, ni pour son stockage : faible spécificité des actifs) ni d'échanges fréquents (transactions peu fréquentes entre producteurs et industriels) : ces facteurs expliquent le fait que le marché soit le principal mécanisme de coordination des acti- vités économiques. Du point de vue politique, le seul levier d'action des producteurs pour se défendre des importations est l'établissement de droits de douane : tout comme le lait, la production de haricot au Costa Rica n'est pas compétitive au regard de celle d'autres pays producteurs.

\section{Défense des intérêts de la filière haricot: les acteurs}

Les producteurs et les industriels du haricot ont des intérêts politiques opposés. Les industriels cherchent à abaisser le droit de douane de façon à s'approvisionner à moindre coût sur le marché international, alors que les producteurs revendiquent un droit de douane élevé pour se protéger des importations. Politiquement, les industriels ont été prompts à s'organiser. En 1995, treize entreprises nouvellement créées, spécialisées dans l'importation et la distribution du haricot mettent en place leur propre organisation professionnelle, la Chambre nationale des industriels de grains (CNIG). La défense des intérêts des producteurs de haricot a été beaucoup plus lente et chaotique à se mettre en place. Elle s'est faite au travers d'un syndicat paysan national, Mesa Nacional Campesina, qui contestait la politique de désengagement de l'État et au travers d'une multitude d'organisations de producteurs de haricot nouvellement créées, dont la vocation était avant tout la recherche de moyens de mise en marché du haricot produit localement. En 1995, les ressources dont disposent les organisations sont très hétérogènes.

D'un côté, la CNIG, bien que récemment créée :

- s'insère dans les réseaux politiques de décision (notamment auprès du ministère du Commerce extérieur), et compte sur l'appui actif de ses treize entreprises ;

- dispose de capacités techniques importantes qui lui permettent d'analyser efficacement la situation économique et d'élaborer des propositions techniques abouties, - jouit d'un financement spécifiquement 
destiné à l'activité de négociation ou d'influence politique.

De l'autre côté, les organisations de producteurs :

- présentent d'importantes difficultés financières ;

- n'ont bien souvent pas les connaissances techniques suffisantes pour défendre précisément leurs positions sur le plan des protections tarifaires ;

- ne disposent pas d'appui suffisant de la part de leurs membres (le haricot n'est qu'une des composantes de systèmes de production où il se trouve souvent dans des proportions réduites) et ce malgré une représentativité potentiellement élevée ;

- ces nouvelles organisations de producteurs continuent à s'orienter préférentiellement vers le ministère de l'Agriculture (qui a pendant longtemps été le seul interlocuteur des producteurs) alors que les décisions se négocient et se prennent sous l'égide du ministère du Commerce extérieur.

\section{Défense des intérêts de la filière haricot: le processus}

La filière haricot se caractérise donc en 1995, du fait d'un passé marqué par l'absence d'interactions entre acteurs économiques de la filière et sphère politique, par l'absence d'espaces spécifiques de dialogue et de négociation. L'absence de relations entre les acteurs concerne surtout les producteurs et leurs organisations. Les organisations citées précédemment vont toutefois chercher à se positionner dans la sphère politique, sans qu'il y ait ni dialogue entre producteurs et industriels ni concertation entre les différentes actions menées par les producteurs. En fait, les actions politiques vont se faire de façon cloisonnée et ne correspondent en aucun cas à la défense d'une vision de secteur comme dans le lait. Le processus qui aboutit à la mise en place d'un droit de douane peut se décrire en trois phases.

- Dans une première phase, les industriels vont travailler à l'élaboration d'une propo- sition politique au sein de la CNIG ; de l'autre côté, Mesa Nacional Campesina va chercher à convaincre quelques producteurs et leurs organisations de la menace que constitue pour eux l'ouverture des marchés, sans que ce travail ne débouche toutefois à la rédaction d'une proposition.

- Au cours de la deuxième phase, les discussions vont s'étendre aux acteurs publics : le ministère de l'Agriculture est mobilisé par une multitude d'organisations de producteurs de façon séparée, alors que la CNIG engage des discussions auprès du ministère du Commerce extérieur, se basant sur sa propre proposition politique.

- La troisième phase enfin correspond à la négociation à proprement parler, qui a lieu au ministère du Commerce extérieur, et se fait sur consultation de la CNIG et du ministère de l'Agriculture.

En 1995, la négociation aboutira à la fixation d'un droit de douane de $1 \%$, largement en faveur des industriels ${ }^{4}$.

Entre 1995 et 2003, le droit de douane a été renégocié vint-trois fois (Salazar, 2003), jusqu'à la fixation en 2003 d'un droit de douane fluctuant (élevé en période de production nationale, bas dans le cas inverse). La situation a donc évolué, notamment du fait de la création, en 2003, d'une Commission nationale du haricot, espace formel de dialogue entre acteurs, qui regroupe à la fois des représentants : des organisations de producteurs multiples, de la CNIG, et du ministère de l'Agriculture. Le dialogue aboutit à la formulation d'une proposition politique mixte, intermédiaire entre les intérêts des industriels et des producteurs, qui sert ensuite de base à la négociation avec le ministère du Commerce extérieur. Dans ce processus, la réévaluation

4. Au cours du processus politique, du fait d'une part de l'absence d'espaces de dialogue entre producteurs et industriels et, d'autre part, de la différence des espaces de discussion mobilisés par les industriels et les producteurs, les antagonismes d'intérêts entre producteurs et industriels n'ont pas généré de réels conflits. 
du droit de douane se fait à la faveur des producteurs : lors de la période de production nationale, ces derniers sont protégés par une barrière tarifaire de l'ordre de $40 \%$. Cette évolution vers une meilleure prise en compte des intérêts des producteurs est liée au fait que les organisations de producteurs ont amélioré leur dotation en ressources (meilleure insertion dans les réseaux, soutien des bases productives) par la mise en place de processus d'apprentissage.

Les configurations fortement contrastées des deux filières mettent en évidence la capacité des organisations à être porteuses de changement institutionnel. L'histoire des interactions entre organisations et État semble être un élément déterminant de l'action politique de ces organisations. Il est impossible de comprendre les ressorts des situations actuelles de négociation sans connaître les trajectoires des organisations et des individus y participant. Les ressources dont disposent les organisations sont du reste directement liées à ces trajectoires : l'accumulation d'expériences a permis aux organisations de renforcer leurs propositions techniques, de s'insérer davantage dans les réseaux, et ainsi de se positionner plus favorablement dans la négociation.

\section{Changement institutionnel Action collective et construction de capacités}

L'entrée du Costa Rica à l'OMC constitue, pour le lait comme pour le haricot, un choc institutionnel vis-à-vis duquel les acteurs des deux filières se sont mobilisés différemment. La comparaison des deux filières met en évidence des capacités différentes d'action collective dans le processus d'élaboration des politiques économiques, avec des répercussions sur la durabilité des filières. À l'instar de la filière haricot, la filière lait a su construire et surtout consolider des mécanismes spécifiques de concertation avec le gouvernement, sur lesquels les organisations s'appuient désormais pour mener à bien leurs actions politiques. Dans cette partie nous reprenons et discutons notre schéma d'analyse à partir des éléments des deux études de cas présentées en cherchant à l'enrichir par quelques réflexions sur les déterminants de la réussite des processus d'action collective.

\section{Les facteurs de réussite : ressources, expérience et apprentissage}

Les éléments empiriques apportés alimentent une réflexion sur l'influence des caractéristiques des organisations sur le comportement de ces organisations dans le processus politique, à même de préciser certaines propositions présentes dans la littérature. Pour ce qui concerne les ressources sur lesquelles s'appuient les organisations, nous vérifions que plus ces ressources sont importantes, plus l'organisation est à même de satisfaire ses objectifs par l'action. Nous sommes en mesure d'affiner ce postulat, qui peut paraître simpliste ou du moins intuitif à première lecture : en effet, si les ressources techniques et financières s'imposent comme des éléments déterminants de l'activité des organisations professionnelles agricoles, il en va différemment des ressources humaines, selon qu' on les considère sous l'angle de l'insertion dans les réseaux (formelle comme informelle, élément déterminant) ou sous l'angle de l'appui des bases productives (élément non déterminant). Concernant ce dernier type de ressource humaine, notre étude montre en effet que les critères proposés par Olson en termes de taille des organisations et d'homogénéité des membres ne permettent pas d'expliquer l'ensemble des situations rencontrées : par exemple, les organisations de producteurs de haricot, étant à la fois de petite taille et composées de membres relativement homogènes, étaient pressenties comme facilement mobilisables pour l'action politique (nos observations montrent le contraire). Pour ce qui concerne l'expérience des organisations, cet élément apparaît primordial à l'interprétation des 
divergences observées : entre filières (lait 1995 versus haricot 1995), et entre périodes (haricot 1995 versus haricot 2005). L'expérience entretient d'importants liens avec les ressources dont disposent les organisations, ce qui va dans le sens de nombreux travaux réalisés portant sur l'apprentissage (Argyris et Schon, 1978 ; Hodgson, 1998 ; March, 1991 ; Schmid, 2004). Les ressources dont disposent actuellement les organisations semblent être le résultat de processus d'apprentissage longs et complexes. En 1995, à l'inverse des organisations de producteurs de haricot en cours de constitution, les organisations de producteurs de lait ont une trajectoire longue d'interactions avec le gouvernement, qui éclaire de façon tout à fait pertinente leur niveau élevé de ressources.

L'observation actuelle des organisations de producteurs de haricot est riche d'enseignements quant au rythme et aux mécanismes de cet apprentissage. Ces organisations sont passées en dix ans de comportements inexpérimentés vis-à-vis du processus politique à des comportements plus actifs et mieux ciblés. Il semble que ce soit à la fois par l'observation du comportement d'autres organisations ${ }^{5}$ (apprentissage indirect de type mimétique) et par l'action même (apprentissage direct de type «learning by doing ») que les organisations de producteurs de haricot ont significativement amélioré leur niveau de ressources, au moins du point de vue humain (mise en place d'un dialogue avec les industriels, mobilisation des producteurs par la discussion) et technique (analyse des enjeux liés à l'ouverture des frontières). Cette évolution permet aux organisations de producteurs de haricot de participer aujour-

5. Ces organisations pouvaient être (i) la CNIG ou encore (ii) des organisations de producteurs d'autres filières, notamment de la filière riz, qui a servi de modèle à la structuration du processus politique propre à la filière haricot, par la création d'un espace formel de dialogue. d'hui de manière plus satisfaisante au processus politique (obtention de mesures publiques d'appui).

\section{Dépendance de sentier}

Les deux études de cas, en soulignant le rôle déterminant de l'expérience, montre que la réussite de l'action collective est tributaire d'une logique de dépendance de sentier. La prise en compte du passé des filières lait et haricot permet de mieux comprendre la situation actuelle de ces filières. Dans cette partie, nous cherchons à dépasser le postulat «l'histoire compte», en proposant une ébauche de réflexion sur les facteurs qui font que l'histoire compte, inspirée des travaux de Pierson (2000). Nous distinguons deux groupes de facteurs : les facteurs technico-économiques d'abord, les facteurs politico-institutionnels ensuite. Parmi les facteurs du premier groupe, la fixité des coûts et les effets de coordination expliquent une certaine continuité dans les phénomènes organisationnels et institutionnels observés.

- Coûts fixes. Nous avons vu que, à l'instar de l'activité haricot, l'activité laitière exigeait un niveau élevé d'investissements, qui se traduit à la fois par une spécialisation des producteurs et par des formes hiérarchiques de coordination des activités économiques. Ces éléments expliquent que les acteurs de la filière lait soient plus prompts à défendre leur activité (coûts de retour élevés), par rapport aux acteurs de la filière haricot, qui peuvent plus facilement se réorienter vers d'autres activités économiques.

- Effets de coordination. La coordination des activités économiques de la filière lait se fait selon des mécanismes bien déterminés, dont le fonctionnement s'est consolidé au cours du temps. Les acteurs contribuent à cette consolidation en fondant leur comportement sur les mécanismes existants (rôle de l'habitude, forte institutionnalisation). À l'inverse, dans la filière haricot, il est difficile de prévoir le comportement des autres 
car les mécanismes économiques d'échanges sont trop récents pour pouvoir être stables (faible institutionnalisation) et les producteurs demeurent peu spécialisés, la production dispersée et l'enjeu économique modeste au niveau de l'économie nationale.

Parmi les facteurs du deuxième groupe, des éléments en termes d'asymétrie de pouvoir et de densité institutionnelle éclairent les dynamiques observées.

- Asymétries de pouvoir. Les institutions impliquent une certaine répartition du pouvoir et des richesses : les acteurs favorisés (industriels dans le cas du haricot) auront tendance à chercher à maintenir leurs positions, rendant difficile et long le travail des acteurs défavorisés (producteurs) qui cherchent un renversement de situation ou du moins une situation moins défavorable.

- Densité institutionnelle. Le jeu des organisations de défense des intérêts s'appuie sur des mécanismes particuliers. Dans le cas du lait, ces mécanismes sont à la fois nombreux et stabilisés (les espaces de dialogue et de négociation sont reconnus de tous, ce qui facilite l'action de la CNPIL); dans le cas du haricot, ils sont peu nombreux et non stabilisés (l'espace de dialogue notamment est encore fragile).

\section{Stabilité des compromis institutionnalisés et durabilité des filières agricoles}

Les éléments empiriques présentés permettent de réfléchir sur les critères d'appréciation de la durabilité. Pour construire notre raisonnement, la durabilité est abordée par ses composantes sociales et économiques, au travers d'une réflexion sur les conditions de maintien des secteurs productifs, or la stabilité des compromis politiques établis semble influencer ces conditions de maintien. La remise en perspective des deux études de cas, sur la période 1995-2005, montre une certaine instabilité des compromis dans le cas du haricot jusqu'en 2003 (renégociation fréquente du droit de douane, jusqu'à l'obtention d'une position commune entre producteurs et industriels) et une forte stabilité des compromis dans le cas du lait. Cette observation indique que la stabilité de la décision politique, qui reflète l'établissement de compromis, est un indicateur de la durabilité des filières. Les filières régies par des mesures politiques stabilisées ${ }^{6}$ sont plus enclines à se maintenir économiquement et socialement.

\section{Conclusion}

Cette réflexion amène à conclure que les producteurs ont des possibilités de négocier leurs conditions de maintien dans un environnement fortement marqué par la libéralisation, mais que la mise en œuvre des processus d'action collective est difficile. Nous avons montré que la prise en compte du passé éclairait pertinemment l'observation des phénomènes contemporains et, en ce sens, que la réussite des processus d'action collective s'inscrivait dans une logique de dépendance de sentier. Toutefois, on ne peut se limiter au seul déterminisme du passé. L'apprentissage individuel et collectif qui forge progressivement les capacités d'action collective des organisations est la voie privilégiée pour dépasser à la fois les asymétries de pouvoir et des dotations historiques en facteurs inégales entre les acteurs. Les changements institutionnels à l'œuvre dans les secteurs agricoles ne sont pas univoques et la libéralisation n'obéit pas à un modèle unique. Elle résulte de rapports de forces entre acteurs que le renforcement des capacités des organisations de producteurs peut contribuer à modifier à travers la formation, l'accès à l'information et les processus d'apprentissage collectif.

6. Cas de la filière lait depuis les années 1960, et de la filière haricot depuis 2003. 


\section{RÉFÉRENCES BIBLIOGRAPHIQUES}

Alston L. (1996). Empirical Work in Institutional Economics: an overview. In L. Alston, T. Eggertsson, D. North (Eds), "Empirical Studies in Institutional Change", Cambridge, Cambridge University Press, p. 25-30.

Argyris C., Schon D. (1978). Organizational Learning: A theory of action perspective. Mass, Addison Wesley.

Arias M.-F. (2004). La agricultura costarricense ante la globalizacion : las nuevas reglas del comercio internacional y su impacto en el agro. San José, CR : Editorial de la Universidad de Costa Rica, Serie Instituto Investigaciones Sociales. Briquel B., Vilain L., Bourdais J.-L., Girardin P., Mouchet C., Viaux P. (2001). La méthode IDEA (Indicateurs de durabilité des exploitations agricoles): une démarche pédagogique. Ingénieries, $\mathrm{n}^{\circ} 25$, p. 29-39.

Buchanan J., Tullock G. (1962). The Calculus of Consent: the logical foundation of constitutional democracy. Indianapolis, Liberty Fund.

Chabaud D., Parthenay C., Perez Y. (2005). Environnement institutionnel et trajectoire des entreprises : une analyse northienne de l'industrie électrique. Management international, p. 65-78.

Cook M., Chaddad F. (2004). Redesigning Cooperative Boundaries: the emergence of new models. American Journal of Agricultural Economics 86, n ${ }^{\circ}$, p. 1249-1253.

Crozier M., Friedberg E. (1977). L'acteur et le système : les contraintes de l'action collective, Paris, Le Seuil.

Friedberg E. (1993). Le pouvoir et la règle : dynamique de l'action organisée, Paris, Le Seuil.

Glachant J., Finon D. (1998). Why is there continued diversity in the European Union's electricity industries? A neoinstitutional analysis. Paris, ISNIE, septembre, p. 20.
Hodgson G. (1998). The Approach of Institutional Economics. Journal of Economic Literature XXXVI, p. 166-192.

Landais E. (1998). Agriculture durable : les fondements d'un nouveau contrat social ? Courrier de l'environnement de l'INRA, $\mathrm{n}^{\circ} 33$, p. 5-22.

Levy B., Spiller P. (1994). The Institutional Foundations of Regulatory Commitment: a comparative analysis of telecommunications regulation. The Journal of Law, economics, and Organization $10, \mathrm{n}^{\circ} 2$, p. 201-245.

March J. (1991). Exploration and Exploitation in Organizational Learning. Organization Science 2, p. 71-87.

McCarthy J., Zald M. (1977). Resource Mobilization and Social Movements: a partial theory. American Journal of Socio$\log y 82, \mathrm{n}^{\circ}$ 6, p. 1212-1241.

Melendez C., Gonzalez L. (1998). Desde hace 50 anos, el dia comienza con DosPinos : historia de la Cooperativa de Productores de Leche RL. San José, Comité de Educacion y Bienestar Social de la Cooperativa de Productores de Leche RL.

Ménard C. (2000). Institutions, Contracts and Organizations. Cheltenham, Edward Elgar.

Montero E. (2004). Comportamiento del precio de la leche fluida al productor y al consumidor en Costa Rica : 1984-2003. Camara Nacional de Productores y Industriales de Leche.

North D. (1990). Institutions, Institutional Change and Economic Performance. Cambridge, Cambridge University Press.

North D. (1993). Institutions and Economic Performance. In U. Maki, B. Gustafsson, C. Knused (Eds), "Rationality, Institutions and Economic Methodology", London, Routledge, p. 242-261. 
North D. (1997). Prologue. In J. Drobak, J. Nye (Eds), "The Frontiers of the New Institutional Economics", San Diego, Academic Press, p. 3-12.

Olson M. (1978). Logique de l'action collective. Paris, Presses Universitaires de France.

Penrose E. (1959). The Theory of Growth of the Firm. Oxford, Basil Blackwell.

Pierson P. (2000). Increasing Returns, Pathdependence, and the Study of Politics. American Political Science Review 94, $\mathrm{n}^{\circ}$ 2, p. 251-267.

Reardon T., Barret C. (2000). Agroindustrialization, Globalization, and International Development: an overview of issues, patterns, and determinants. Agricultural Economics 23, n 3, p. 195-205.

Ruiz W. (2002). Politicas de gobierno de apoyo a la produccion de frijol. San José, CR, Ministerio de Agricultura y Ganaderia, p. 1-2.

Salazar J. (2003). Desempeño de la Actividad de Frijol en Costa Rica: Programa Nacional de Frijol. Santo Domingo de Heredia, CR.
Salazar J. (2000). Efecto de la apertura comercial en la produccion nacional de frijol. San José, CR, Ministerio de Agricultura y Ganaderia, p. 21-28.

Salazar J. (2002). La actividad de frijol en Costa Rica en el ciclo 2001-2002. San José, CR, Ministerio de Agricultura y Ganaderia, p. 7-16.

Schmid A. (2004). Conflict and Cooperation: institutional and behavioral economics. Oxford, Blackwell Publishing. SEPSA (2002). Desempeno de la ganaderia de leche y de la industria de la transformacion de productos lacteos en Costa Rica : 1996-2001. San José, Ministerio de Agricultura y Ganaderia.

Shirley M. (2004). Institutions and Development. Tucson, p. 44.

Stigler G. (1971). The Theory of Regulation. Bell Journal of Economic and Management Science, $\mathrm{n}^{\circ}$ 2, p 3-21.

Wernerfelt B. (1984). A Resource-Based View of the Firm. Strategic Management Journal 5, n 1, p. 171-180. 